\title{
USE OF SAR SATELLITE DATA IN BRIDGE MONITORING WITH APPLICATION TO URBAN AREAS
}

\author{
Luca Sartorelli ${ }^{1}$, Mattia Previtali ${ }^{2[0000-0003-4588-9045]}$, Pier Francesco Giordano ${ }^{3[0000-0003-}$ \\ ${ }^{0396-0253]}$, Maria Pina Limongelli ${ }^{40000-0002-9353-5439]}$, Francesco Ballio ${ }^{5[0000-0002-8499-1707]}$ \\ and Andrea Uttini ${ }^{6}$ \\ ${ }^{1}$ Politecnico di Milano, Milan, Italy \\ ${ }^{2-4}$ Department of Architecture, Built Environment and Construction Engineering, Politecnico \\ di Milano, Milan, Italy \\ ${ }^{5}$ Department of Civil and Environmental Engineering, Politecnico di Milano, Milan, Italy \\ ${ }^{6}$ Tre Altamira, Milan, Italy \\ mattia.previtaliepolimi.it
}

\begin{abstract}
In the last 20 years, SAR sensors installed on satellites have been increasingly used to monitor large scale phenomena such as subsidence, uplifting, or landslides. Recently several research groups started to investigate the use of remote sensing for damage detection in civil infrastructures. This technique presents in principle several advantages, not least the possibility to monitor a network of several structures using the same sensor. However, its implementation for civil engineering projects is still at its infancy and a limited number of applications can be found in literature. The goal of this paper is to investigate the feasibility of using InSAR data for structural health monitoring of bridges with reference to a number of case studies. Data measured by radar satellites with different spatial and temporal resolutions and different frequency bands are processed to extract bridge displacement data and analyzed to identify possible structural damages. The SqueeSAR technique is used to extract displacement time histories from SAR images acquired by radar sensors with different spatial and temporal resolution (TerraSAR-X and RADARSAT 1-2). Displacements are then processed to identify anomalies possibly related to damage. Results show that highresolution radar data enable the detection of very small structural displacements due to environmental changes, such as temperature variation.
\end{abstract}

Keywords: Satellite SAR Remote Sensing, Bridge Health Monitoring, Data Processing, SqueeSAR. 


\section{Introduction}

Bridges, which are critical elements of transportation networks, are subjected to several degradation phenomena, such as aging, fatigue, and chemical attacks. Insufficient maintenance interventions, joined with increased traffic loads with respect to the design values as well as effects connected with climate change, often accelerate materials deterioration. Over the last decades, global warming has led to a growth in intensity, frequency, and duration of extreme natural events that exacerbate the effect of deterioration, often leading to conditions not accounted for in the original design standard [1]. Currently, a large percentage of road and railway bridges in Europe needs urgent rehabilitation due to structural damages. A considerable number of Italian infrastructures were designed and constructed over 50 years ago and they need maintenance to avoid serious and dramatic consequences. It has been estimated [2] that tens of billions of euro are needed to preserve the Italian bridges' health.

Visual inspections are traditionally used to collect information about the bridge conditions and to support maintenance planning. These operations are time-consuming, strongly subjective, and above all, they do not provide real time information able to support prompt decisions for maintenance management. To fill this gap, in the last two decades, Structural Health Monitoring (SHM) techniques, based on the extraction of information through the processing of data collected by sensors, have been developed [3]-[6]. Traditional sensors are installed on the monitored structure to record its response. However, the acquisition and processing of data can lead to high costs that network owners may not be available to or cannot sustain.

In the last 20 years, due to technological advances in radar sensing and data processing, Synthetic Aperture Radar (SAR) satellite data have been increasingly used for monitor purposes in earth sciences. In particular, Interferometry Synthetic Aperture Radar (InSAR) is a remote sensing technique that allows to detect displacements with subcentimetric accuracy over large areas. The InSAR feasibility to measure displacements related to large-scale events such as landslides or subsidence has been assessed for the past two decades. Recently, with the increase of radar spatial resolution, the use of this technique to monitor displacements of civil structures, such as bridges, has become feasible.

The goal of this paper is to report some preliminary results of an investigation aimed to assess the possibility to use SAR data to monitor the evolution of the structural health of bridges. In particular, a procedure to extract information from satellite radar data is presented with reference to several case studies in the Milan urban area. Interferometric data acquired by high and low spatial resolution sensing devices - namely TerraSAR$\mathrm{X}$ and RadarSAT-1/2 - are used to extract displacement and to verify the capabilities of the two types of sensors. InSAR data are processed using the multi-interferogram SqueeSAR® technique [7].

The paper is structured as follows. Section 2 contains a short literature review analysis on the application of InSAR monitoring to bridges. Section 3 briefly presents the SqueeSAR technique and the features of two sensing systems and of the data used in the paper. Section 4 describes the methodology implemented to extract preliminary information about the bridge condition from displacement time series. The case studies 
and the discussion of results are described in Section 5 and Section 7 concludes the paper.

\section{Related work on the use of InSAR for bridges}

In the last decades, the InSAR technique has been increasingly used to monitor large scale phenomena such as subsidence, uplifting, or landslides [8]-[11]. Recently, thanks to the improved spatial resolution of radar satellites, its use to monitor civil structures has been developing, leading to several applications. One of the main advantages of remote sensing is that it can offer a look back in time over the study area. In several papers, it is highlighted how the analysis of InSAR displacements could have provided useful information about impending structural failures months before the collapses. Sousa and Bastos [12] showed that the use of multi-temporal InSAR techniques might have foreseen the collapse of the Hintze Ribeiro centennial bridge in Portugal. Based on this observation the authors propose an early warning system triggered by the exceedance of a displacement alarm threshold. Selvakumaran et al. [13] showed that the partial collapse of the Tadcaster Bridge due to scour could have been predicted within one month by an automatic outliers identification method. Recently, Milillo et al. [14] studied the evolution of the configuration of the Morandi bridge over a period of about 15 years before the failure, although their results have been questioned by other authors [15].

SAR satellites have a constant revisiting time ranging from weeks to months thereby they can be used to follow the evolution of the structural condition during all life phases of a bridge, from the design to the construction and operational phases. Pigorini et al. [16]. showed as Persistent Scatters InSAR (PSInSAR) could have been applied in the preliminary design of the new Venice-Trieste railway to study the behavior of the study area. In Pigorini et al. [16] and Koudogbo et al. [17], InSAR is used as a monitoring survey during the construction and operational phases of the Scianina-Tracoccia tunnel and Grand Paris Express metro network respectively.

Several authors highlighted that the effect of environmental factors in InSAR displacements monitoring must be properly taken into account to avoid false or missing indications when these data are used to assess the structural health. Besides, InSAR data can be used to study the effect of environmental factors (such as temperature and water action) on a specific infrastructure.

In literature, the correlation between temperature and displacements measured by SAR data at different radar bands ( $\mathrm{C}$ and $\mathrm{X}$ ) has been extensively investigated. Both Fornaro et al. [18] and Lazecky et al. [19] demonstrated the high level of correlation between temperature and displacement acquired by X-band, while Huang et al. [20] studied the correlation between displacement from C-band scenes and temperature for the metal Nanjing Dashengguan Yangtze River bridge. Other authors studied the correlation between InSAR displacements and other environmental conditions. In References [12], [13], the authors highlighted that SAR can detect changes correlated to water actions, such as scour and changes in flow rates during flooding, in riverine bridges. Zhang et al. [21] used SAR to study the performance of the Donghai bridge, which is 
located in a very rough sea environment, and suggested that the InSAR displacements could be correlated to seasonal winds and tide. Another phenomenon that might be studied using SAR data is bridge settlements due to soil consolidation, as presented by Del Soldato et al. [22].

The effect of environmental factors on bridge displacements may hinder the correct identification of damage if not properly accounted for. In this respect, Huang et al. [23] developed a damaged detection method based on the removal of the thermal effect from the detected displacements.

The comparison between information provided by satellite images and in-situ data is an important factor to prove the InSAR capability in structural damage detection. Despite a large number of civil structures such as dams [24]-[26], buildings [27], and historical monuments [28] monitored using InSAR techniques, the validation of results using data collected directly on bridges is seldom performed, as highlighted by Cusson et al. [29].

\section{Extraction of bridge displacements from InSAR images}

In this section, a brief description of the interferometric technology is presented to make the paper self-contained.

SAR is a radar satellite measurement system that provides images stored as matrices of complex numbers that represent amplitude and phase values of the reflected radar signal. Interferometry is the technique that is used to derive displacement time series from the radar data by detecting phase differences through time [17]. Differential interferometric SAR (DInSAR) relies on the processing of two SAR images acquired over the same area at different times, using the same acquisition geometry, and measures possible phase variations induced by surface displacements [27]. The main drawback of DInSAR is that phase differences may not only be induced by displacements. Indeed, temporal and geometric decorrelation phenomena, as well as atmospheric effects may have an impact on the signal phase, thereby reducing the quality of DInSAR results. In the late nineties, new, multi-interferometric, techniques were developed, aiming at identifying individual pixels exhibiting stable radar returns (now referred to as Persistent or Permanent Scatterers - PS). Multi-image algorithms can improve significantly any DInSAR analysis, allowing one to increase the sensitivity and the quality of the results. InSAR requires the processing of long time series to remove the atmospheric component which varies slowly in space and is not correlated in time. Persistent Scatterer InSAR (PSInSAR) was the first multi-temporal technique exploiting pointwise PS [30]. About ten years later, to overcome the limits of PSInSAR, mainly related to the low PS density in non-urban areas, a new multi-interferogram InSAR algorithm was developed [7]. This new technique allows one to identify Measurement Points (MP) belonging to two groups: i.e. Persistent Scatterers (PS) and Distributed Scatterers (DS). The PS are discrete point-wise radar targets characterised by high signal-to-noise values and stable radar signature (i.e. high reflectivity and coherent phase values) in time. PS are slightly affected by temporal and geometric decorrelation as is the case of points lying on buildings, poles, rocky outcrops and man-made structures. The DSs are 
patches of ground exhibiting lower but homogenous and consistent radar returns. DSs can be exploited only if they form sufficiently large homogeneous groups of pixels sharing the same scattering mechanism; in these conditions statistical analysis can be used to reduce noise effects.

SqueeSAR needs a dataset of at least 15-20 SAR images, acquired over the same area with the same acquisition mode and geometry, to identify MPs on the Earth surface. For each MP, this technique provides the ground target's position (in WGS84 coordinates), the ellipsoidal elevation (referred to the ellipsoid WGS84), the annual average displacement rate along the Line-of-Sight (LOS) and its standard deviation (expressed in $\mathrm{mm} / \mathrm{year}$ ), displacement time series (in $\mathrm{mm}$ ) measured along the LOS, and other MP features, such as coherence and average seasonal components. The precision of SqueeSAR displacement measurements mainly depends on the quality of the processed dataset and of the estimation of the atmospheric contributions. The former relies on: the number of processed SAR images, the length of the period time analysed, temporal continuity of acquisition and correctness of phase unwrapping. The atmospheric noise intensity depends on the tropospheric conditions at the time of the acquisitions, the acquisition geometry and the distance from a reference point (REF) used for calibration [25]. Overall, SqueeSAR has an accuracy of about $1 \mathrm{~mm} / \mathrm{year}$ and a precision for the single measurement around $5 \mathrm{~mm}$, for a MP located less than $1 \mathrm{~km}$ away from the REF and considering a dataset of at least 30 SAR images covering 2year period [7].

The data used for the investigation reported in this paper are acquired by RadarSAT1/2 (RSAT) and TerraSAR-X (TSX) and processed through SqueeSAR. The features of the dataset are reported in Table 1.

Table 1. Comparison between two datasets: TerraSAR-X and RadarSAT-1/2.

\begin{tabular}{ccc}
\hline & TerraSAR-X & RadarSAT-1/2 \\
\hline Space Agency & DLR (Germany) & CSA (Canada) \\
Radar Band & $\mathrm{X}$ & $\mathrm{C}$ \\
Wavelength $\lambda[\mathrm{cm}]$ & 3.11 & 5.66 (RSAT1) -5.55 (RSAT2) \\
Spatial resolution $\left[\mathrm{m}^{2}\right]$ & $3 \times 3$ & $20 \times 5$ \\
Elevation precision* $[\mathrm{m}]$ & \pm 1.5 & \pm 1.5 \\
Revisiting time $[$ day $]$ & 11 & 24 \\
Acquisition period & $31 / 08 / 2009-23 / 02 / 2020$ & $13 / 10 / 2003-23 / 10 / 2017$ \\
$\mathrm{~N}^{\circ}$ images & 267 & 198 \\
Satellite geometry & Descending & Descending \\
Sensor Mode & SM010 (StripMap) & S3 (Standard) \\
\hline
\end{tabular}

*Typical precision value of MP, located less than $1 \mathrm{~km}$ from the REF and a dataset of at least 30 SAR images, associated with the UTM coordinate of MP at mid-latitudes.

For both datasets, the displacement time series and annual displacement rate are measured along the LOS of the descending orbit trajectory. Since one satellite geometry is used in this paper (namely the descending one), it has not been possible to retrieve 
the displacement components in vertical and horizontal (East-West) directions [31]. Hence, a negative displacement corresponds to MP movement away from the satellite, while a positive one is directed towards the satellite.

\section{$4 \quad$ Analyses of displacement data}

This section describes the procedure adopted to process the LOS displacements provided by the SqueeSAR technique and to extract preliminary information about the bridge health. The workflow represented in Figure 1 was followed.

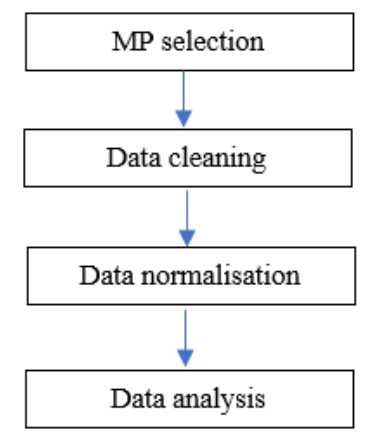

Figure 1. Analysis of displacement data workflow.

Firstly, MP selection is carried out by using the software QGIS Desktop 3.10.6 [32] that allows one to superimpose the MPs to the orthophoto of the study area, downloaded by Geoportale Regione Lombardia [33]. The MPs located on the bridge deck are selected and furtherly processed to infer bridge displacements and preliminary information about the bridge's health. The remaining MPs are used to investigate the displacements affecting the area near the bridge.

The second step consists in data cleaning which is applied to each bridge dataset to identify possible outliers and remove them from the datasets used for further processing. Two types of outliers are considered, namely: MPs erroneously located on the bridge deck due to inaccuracies in the overlap of the SqueeSAR outcomes to the orthophoto, and MPs exhibiting anomalous values of the annual displacement rate. The first type of MPs is identified by comparing their elevation with the elevation of the bridge deck and accounting for the positioning accuracy of the two satellites (Table 1). For example, the limits shown in Figure 2, which are related to the Via Lodovico il Moro bridge 2 (see Section 5.2), correspond to the lower and upper elevation of the bridge deck above the WGS84 ellipsoid (between 154 and $162 \mathrm{~m}$ ) considering the precision of satellite radar. The second type of outliers are those with values of the displacement rate outside the confidence interval of two standard deviations relevant to the dataset including the annual displacement rate of all the $\mathrm{MP}_{\mathrm{S}}$ located on the bridge. Under the assumption that the MP displacements are normally distributed, this assumption corresponds to a probability of $5 \%$ to wrongly discard an MP that is actually located on the bridge. 


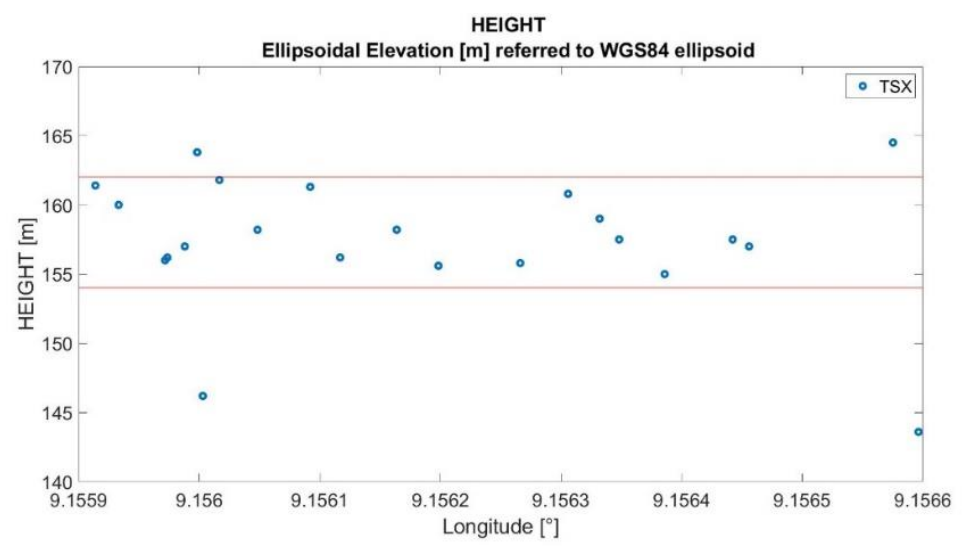

Figure 2. An example of detection of the first type outlier for Via Lodovico il Moro bridge 2. Red lines are the bridge elevation limits, so the dots out of the red lines are considered outliers.

In the following data normalization phase, the two datasets are normalized to allow comparison. Indeed, the two datasets, TSX and RSAT, have different acquisition times (see Table 1). To allow the comparison of datasets, an overlapping period is selected (RSAT-1/2: 18/08/2009 - 23/10/2017 and TSX: 31/08/2009 - 18/10/2017), and the mean of the displacements measured during the first two-years is subtracted to the displacement time series of each MP. Moreover, a moving average over 4 months of the displacement time series is applied to filter some oscillations due to noise.

Finally, data analysis is carried out to detect trends and patterns that may provide preliminary information about bridge health. This analysis entails several operations on the displacements dataset, as follows:

a. Identification of regions of the bridge deck that may be assumed to behave as rigid bodies and relevant clustering of the dataset. These regions can be identified based on the analysis of the geometry of the bridge such as, for example, influence zones of the piers or regions separated by expansion joints. More details will be given in the section of the case studies.

b. Identification of a Representative Displacement (RD) for each cluster of data. In the analysis reported in this paper, the mean displacement computed for each epoch over the cluster MP displacements is assumed as RD.

c. Comparison of the RD of each cluster with the displacements of the area surrounding the bridge. This is aimed to discard in the damage detection analysis large scale displacements that affect the entire region surrounding the bridge, thereby causing only rigid body motions of the entire structure.

d. Analysis of the correlation between RDs and environmental changes (i.e. temperature). This is performed through the analysis in the frequency domain of the displacement time history and the computation of the Pearson correlation coefficient between the displacement and the temperature time histories.

e. Validation of the trends identified in the previous step through information collected on-site or through documents and reports relevant to maintenance interventions performed on the bridge. 


\section{Case studies: railway and road bridges in Milan}

The procedure outlined in the previous section was applied to several bridges in the Milan urban area crossed by a wide transportation network of roads, highways, and railway. One of the aims of the analysis was to compare interferometric data acquired by sensors with different spatial resolution. Data acquired by TerraSAR-X (high resolution) and RadarSAT-1/2 (low resolution) were available for slightly different portions of the Milan urban area, thereby the analysis was carried out only for bridges located in the overlapping region between the tiles relevant to the two satellites. Bridges spanning more than $20 \mathrm{~m}$ were selected since the RSAT spatial resolution is limited to about $20 \times 5 \mathrm{~m}^{2}$. So, bridges with a span smaller than 20 may result in having a MPs density insufficient for a fair and meaningful comparison with TerraSAR-X. In this paper, it is reported and discussed the results obtained for four bridges, detailed in Table 2 and Figure 3.

Table 2. Studied bridges.

\begin{tabular}{ccccc}
\hline Bridge & Long. $\left[{ }^{\circ}\right]$ & Lat. $\left[{ }^{\circ}\right]$ & Length $[\mathrm{m}]$ & Type \\
\hline Via Farini & 9.182400 & 45.487175 & 150 & Vehicular + Tramvia \\
Viale Monza 101 & 9.219933 & 45.497004 & 26 & Railway \\
Viale Monza 119 & 9.220472 & 45.498395 & 28 & Railway \\
Via Lodovico il Moro 2 & 9.1566659 & 45.447836 & 45 & Railway \\
\hline
\end{tabular}

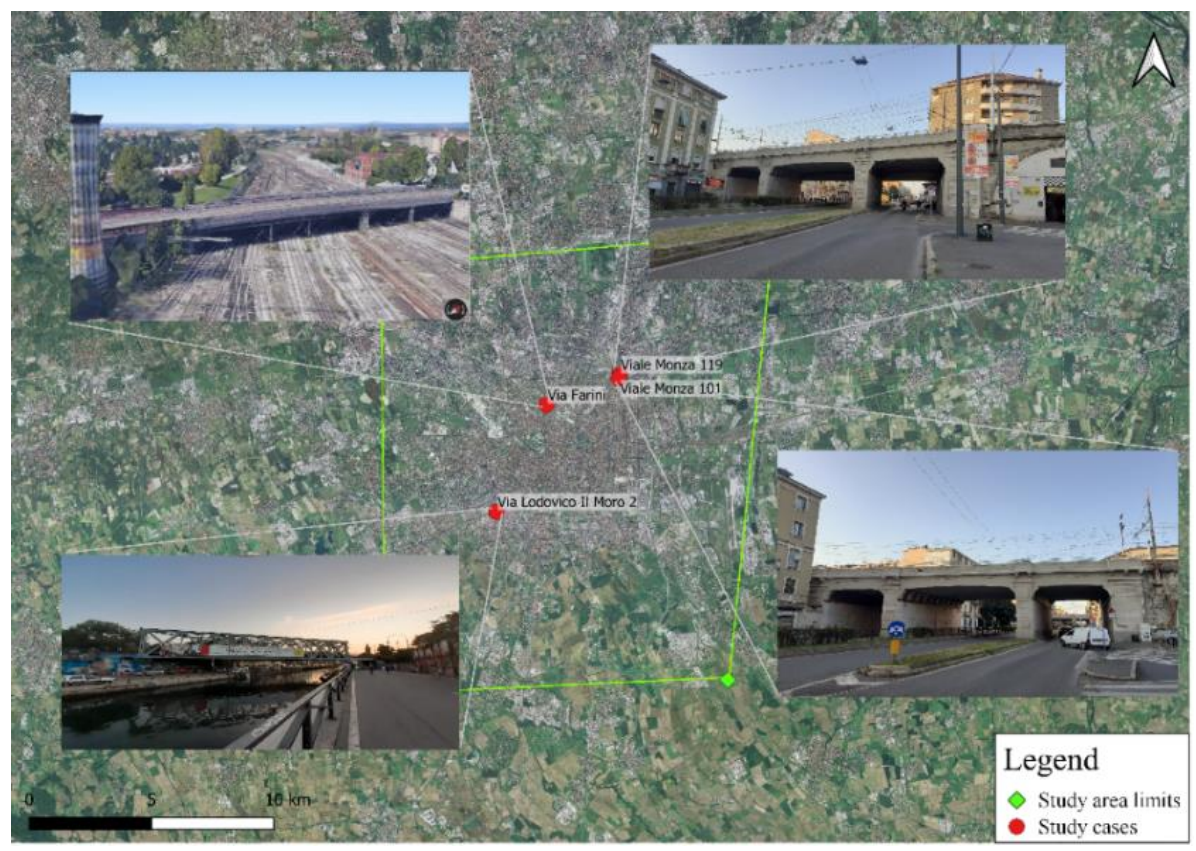

Figure 3. Location of the case studies. 


\subsection{Large scale analysis of the Milan urban area}

The large-scale analysis of the study area highlighted a peculiar trend of the LOS displacements measured along the railway infrastructure. These displacements are all directed away from the satellite highlighting a global settlement of this infrastructure. This is very clearly shown by Figure 4, where the annual displacement rates along the LOS are reported for the considered area: The location of the MPs with higher velocity follows exactly the path of the railway. These displacements are attributed to gradual settlement of railway ballast due to repeated loading cycles during the usage.

Figure 5 displays the average displacement of the MPs located on the railway in proximity of the Lodovico il Moro 2 and the two Viale Monza bridges. Indeed, in it is evident that railway areas are subject to a decreasing trend in the displacement time series. During the analysis of InSAR data, these displacements might be wrongly interpreted as structural displacements and therefore must be carefully accounted for. Herein, in order to remove this possible source of errors in the study of the bridge condition, the MPs located in proximity of the railway are not considered in the analysis.

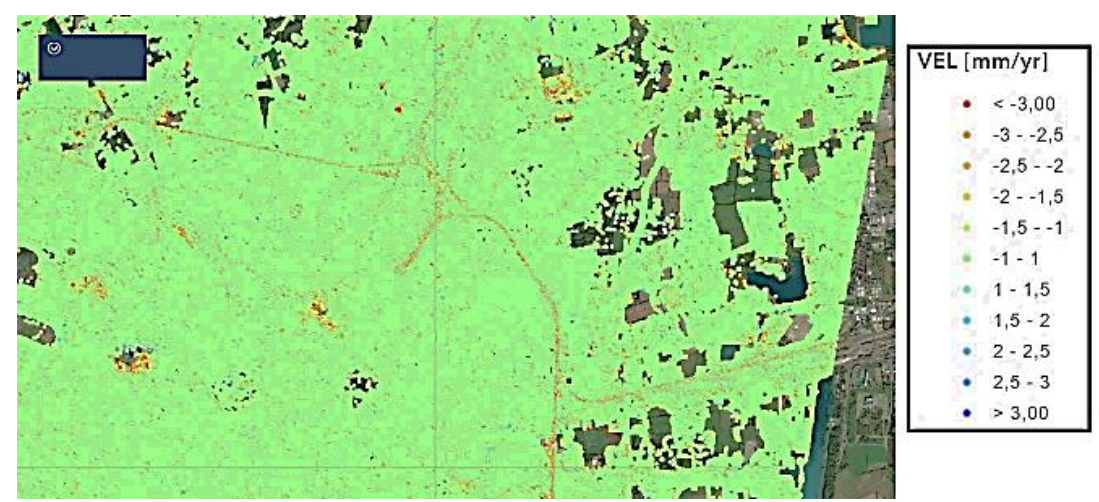

Figure 4. Annual displacement rate along LOS (descending trajectory). Long red lines, made by MPs, are observable. They correspond to railway tracks.

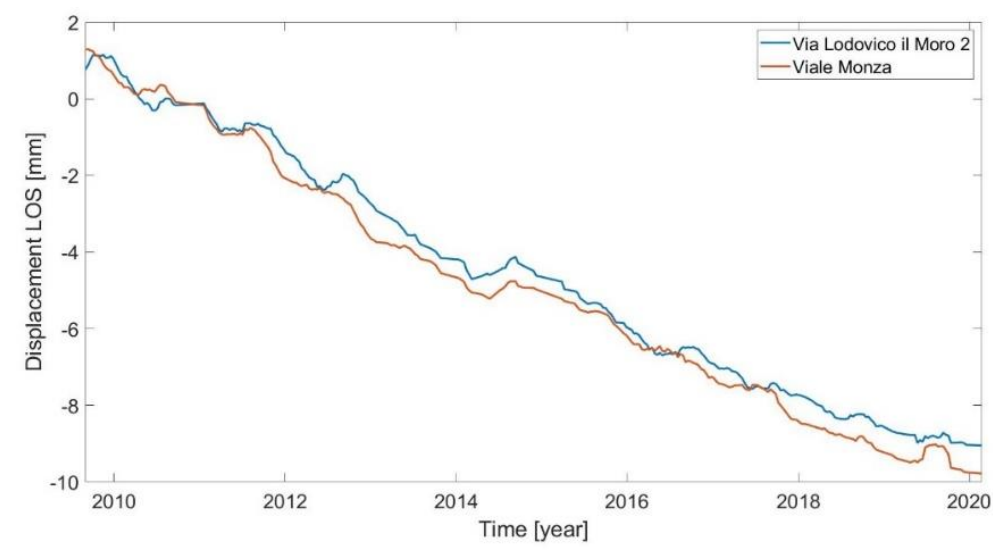

Figure 5. The mean displacement between MPs belonging to the railway areas in proximity of Via Lodovico il Moro 2 and the two Viale Monza bridges. 


\subsection{Via Lodovico il Moro 2 bridge}

Via Lodovico il Moro 2 bridge crosses the Channel Alzaia Naviglio Grande and it is one of the longest metal railway bridges in the study area. It is a steel truss bridge that lays on two lateral concrete piers.

The analysis of bridge displacements was carried out using the two available datasets, RSAT and TSX. The MPs detected on the bridge by the two satellites are visible in Figure 6. In this case, all the MPs were supposed to be part of the same cluster and the average displacement was considered as the reference displacement for the bridge.

Figure 7 reports the RDs retrieved from TSX and RSAT data. Both time series oscillate around the zero displacement and show a certain periodicity.

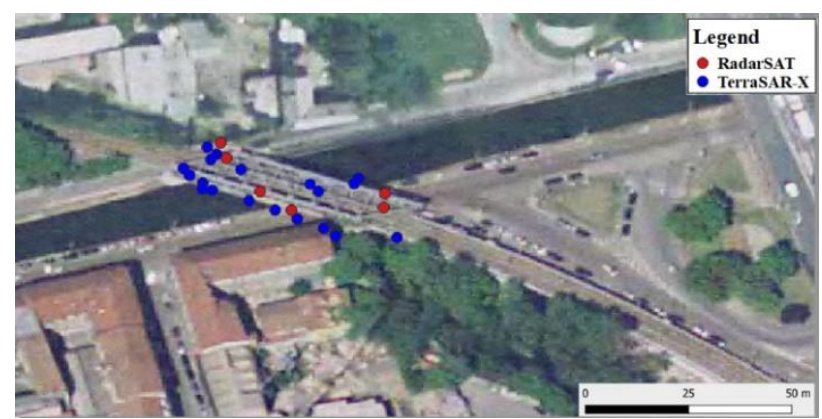

Figure 6. Via Lodovico il Moro 2: MPs on the bridge acquired by TSX and RSAT

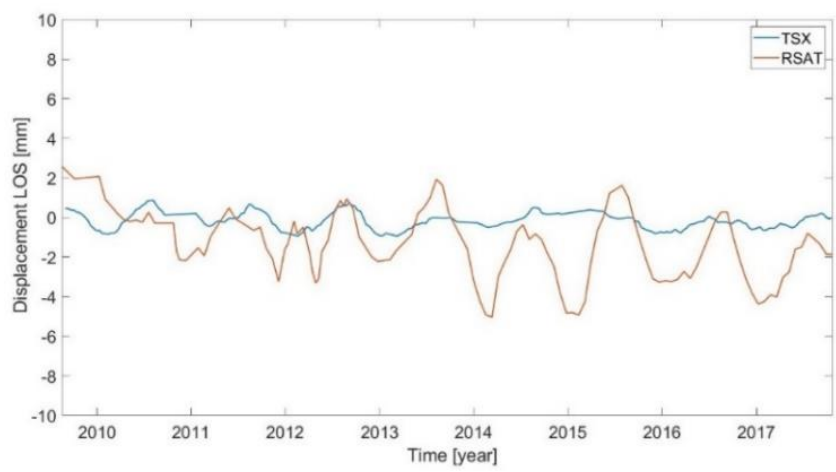

Figure 7. Via Lodovico il Moro 2 bridge: Comparison between RDs time series provided by TSX and RSAT.

The correlation coefficient between RSAT and TSX displacements is equal to 0.43 which indicates a low but not negligible correlation between the two displacement datasets. However, the magnitude of the displacements of the two datasets are quite different. This difference is likely due to the different sensitivity - related to the different radar wavelength and to possible cycle slips, i.e. discontinuity in radar phase - of the two sensors to detect a phase change. The ratio between the mean values of displacement measured by the two satellites is (on average) equal to 2 , a value which is close 
to the ratio of 1.8 between RSAT and TSX wavelengths. Of course, it should be pointed out that the two sensors have different acquisition geometries and different radar parameters; therefore, PS from the two data-sets can correspond to different objects on ground.

For all the case studies considered, the correlation between the displacement and the minimum daily temperature [34] is investigated. The minimum value is considered for the daily temperature because both satellites pass over the study area at around 05:30AM, when temperature values are at their minimum.

Figure 8 reports the time histories of the temperature and of the displacement extracted from the two datasets. The Pearson correlation coefficient [35] between the two time series is respectively 0.68 and 0.60 for TSX and RSAT. This trend highlights the strong correlation between the two series indicating that for this bridge the structural displacements are mainly due to thermal variations. A further indication is given by the displacement Fourier spectra (see Figure 9. ) which presents a sharp peak at the yearly frequency of the temperature cycle.

The comparison between the displacements of the bridge and those of the surrounding area show that there are not significant relative displacements between the two. The bridge displacements are mainly due to thermal variations thereby structural damages do not seem to have occurred in the observation period.

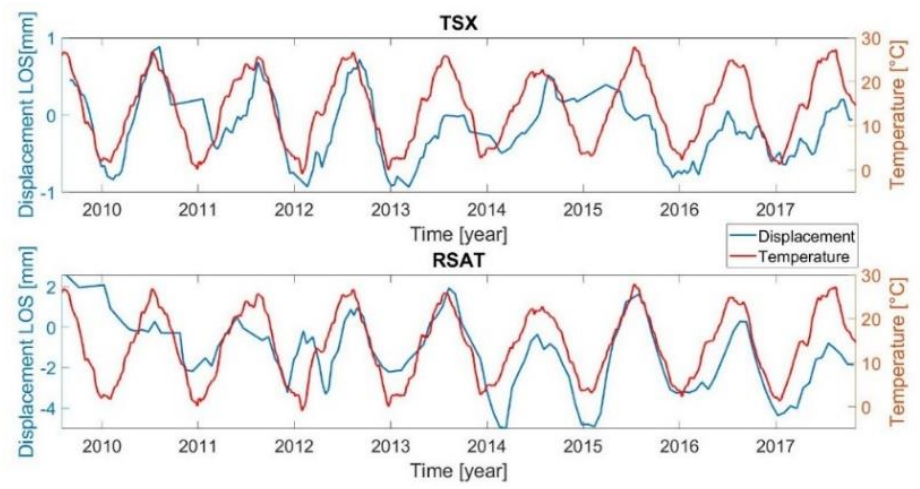

Figure 8. Via Lodovico il Moro 2 bridge: Representative Displacement (blue) and temperature (red) time series for TSX (above) and RSAT (below) datasets.

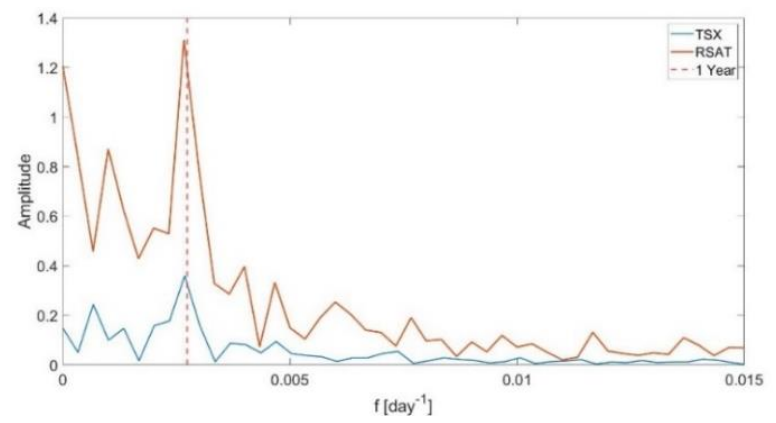

Figure 9. Via Lodovico il Moro 2 bridge: Fourier spectra of displacement time series. 


\subsection{Via Farini bridge}

Via Farini bridge spans around $200 \mathrm{~m}$ with a hyperbolic profile. The southern span is simply supported on the piers whereas the northern spans form a continuous beam on three supports. The MPs provided by both the TSX and the RSAT are all located on the eastern and western sides of the deck thereby ten MPs clusters were identified on the regions delimited by four transversal and the longitudinal central joints (see Figure 10a). Since the RSAT dataset contains a lower number of MPs with respect to the TSX dataset (as should be expected), their comparison was possible only on the six clusters represented in Figure 10b by the hatched areas. For this case study, the data retrived by the two different satellites are not comparable in terms of displacement magnitude, as highlighted in Figure 11.

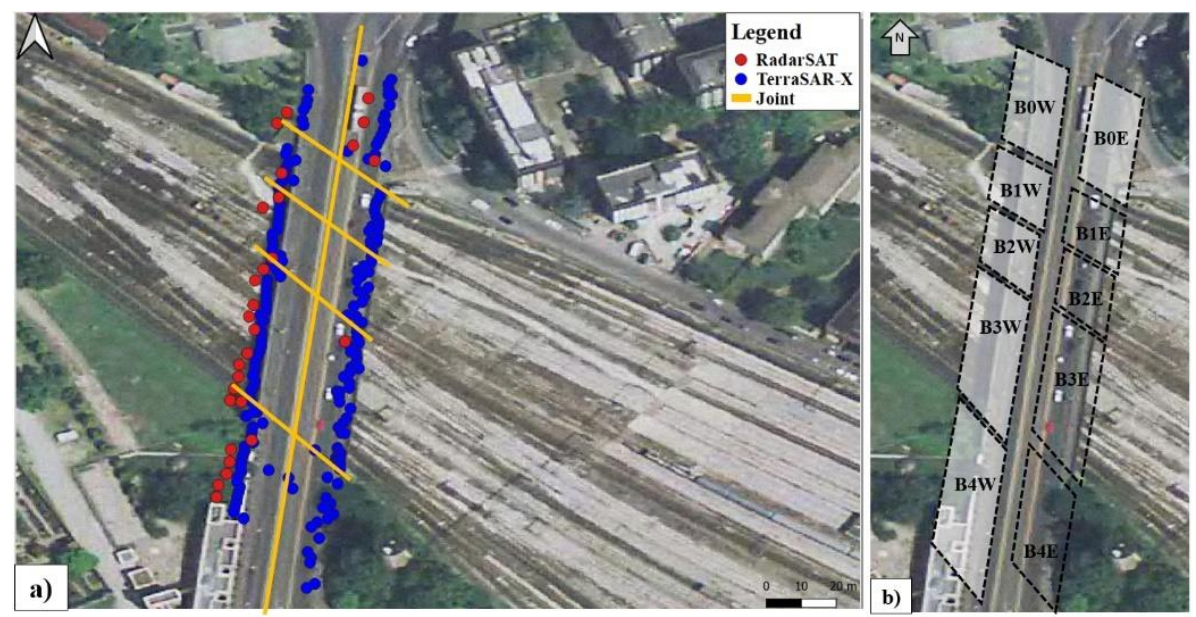

Figure 10. Via Farini bridge: a) MPs detected (before the data cleaning) by TerraSAR-X in blue and RadarSAT in red, separated by the joints in yellow; b) MP clusters: Hatched areas where both TerraSAR-X and RadarSAT MPs are detected.

The Pearson correlation coefficients between the temperature and the RDs extracted from the TSX dataset are shown in Figure 12 for each of the 10 clusters. The dataset is relevant to the period between September 2009 and February 2020 when maintenance works were initiated on the bridge. For the blocks showing higher coefficients $\rho$, also the linear regression line is displayed.

The analysis of these datasets shows (see Figure 12) that the displacements of the central blocks (indicated as B1E, B2E, B3E, B2W, and B3W in Figure 10b) are linearly correlated with the temperature, while very low values of correlation is shown by the displacements of the clusters close to the abutments (B0E, B0W, B4W and B4E, and $\mathrm{B} 1 \mathrm{~W})$. The RDs of the central-eastern blocks (1E, 2E, 3E) have a positive correlation with the temperature, while the central-western blocks have a negative correlation. This displacement pattern, with displacements of central-eastern blocks specular with respect to those of the central-western side, is consistent with the longitudinal expansion and shrinking at the longitudinal central joint, induced by thermal variations. 
The eastern and western sides of the three blocks B0, B1 and B3 move in opposite directions in time (Figure 13). Furthermore, from the maintenance reports, it is noticed that these blocks were recently impacted by important maintenance works such as enlargement of the loading piers, reinforcement of the abutment, and strengthening of the supporting structures. The anomalous behaviour of the blocks and the fact that maintenance works have been carried out leads to suppose that the displacement trends were due to damage.
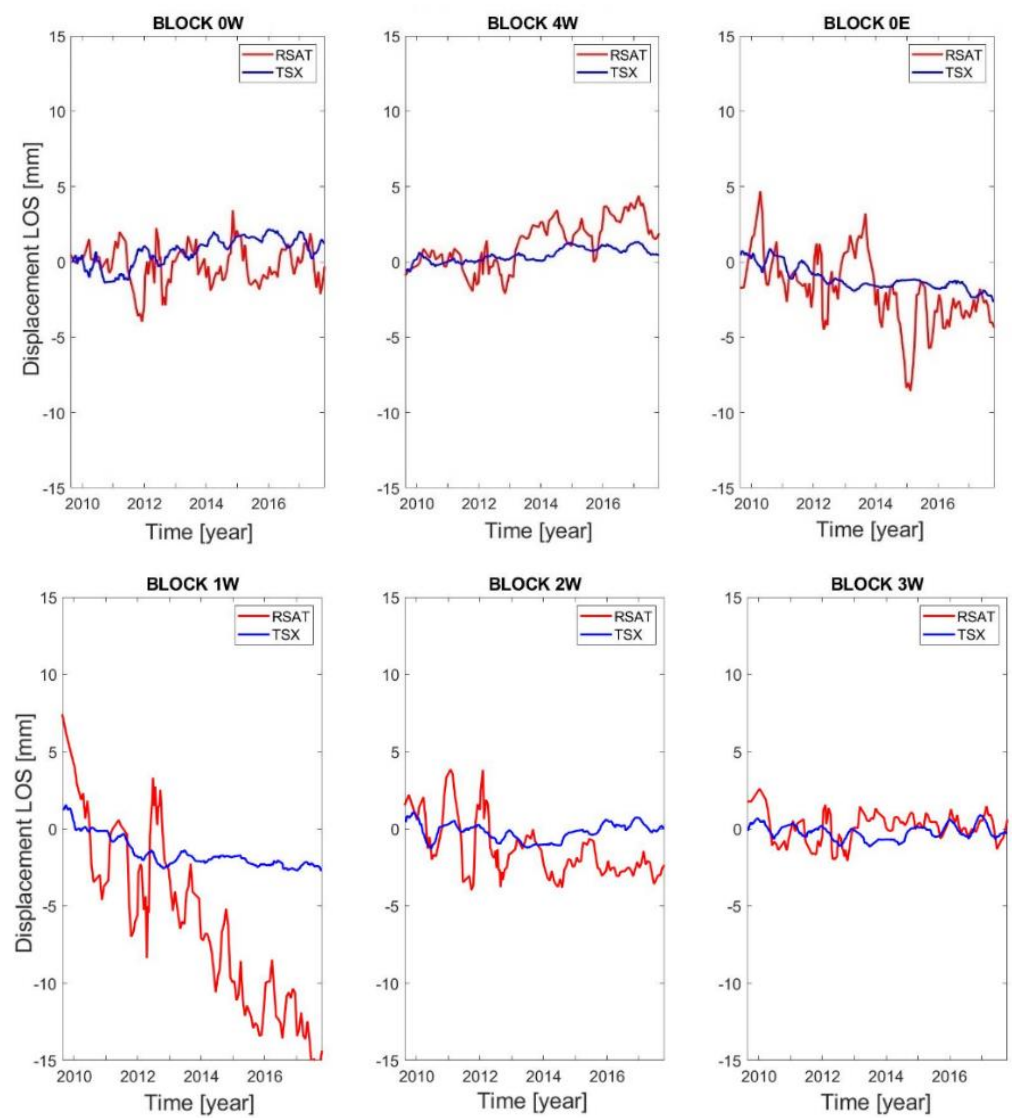

Figure 11. Comparison between RD of TSX and RSAT datasets for the 10 clusters. 

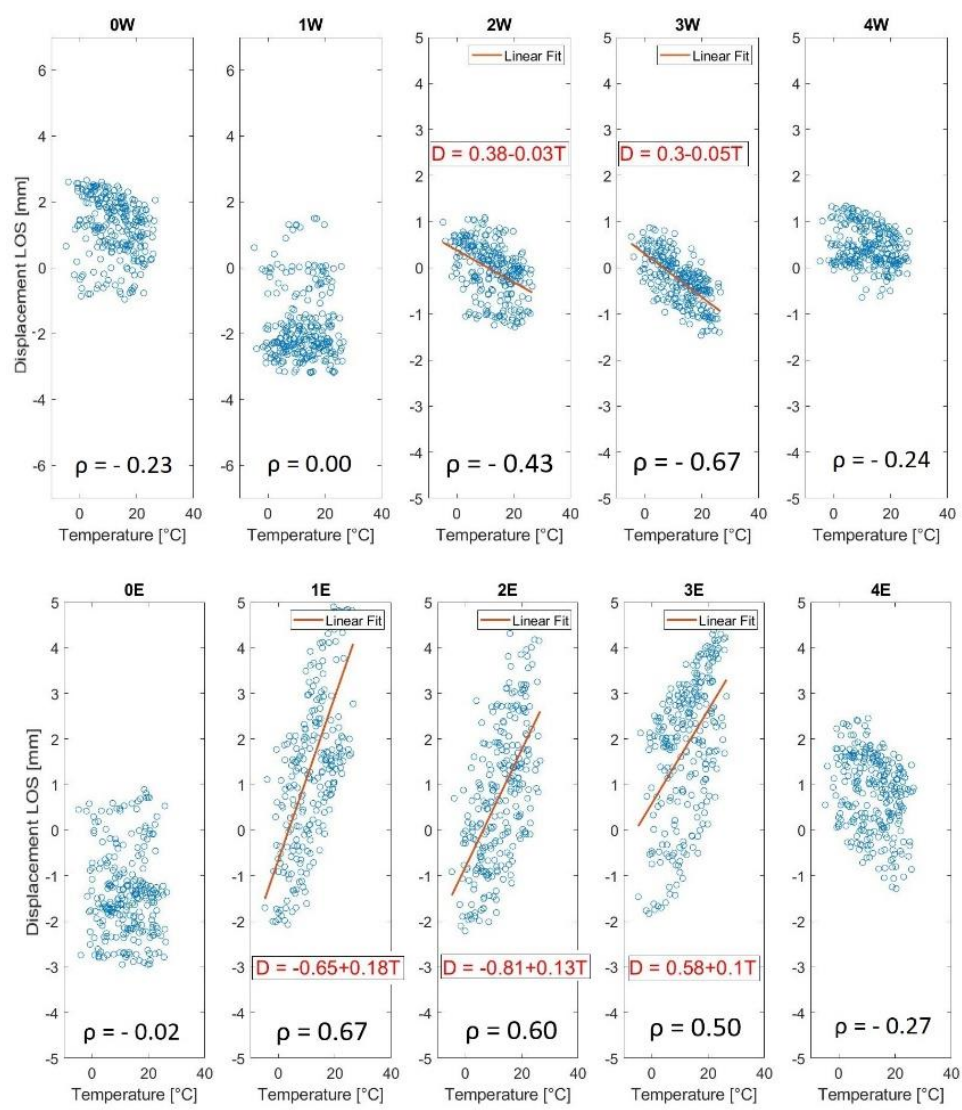

Figure 12. Via Farini bridge: TRX displacements VS minimum daily temperature plots and Pearson correlation coefficients $(\rho)$ for all TSX blocks. For the blocks showing higher coefficients $\rho$, the linear regression line $\mathrm{D}(\mathrm{T})$ is displayed.
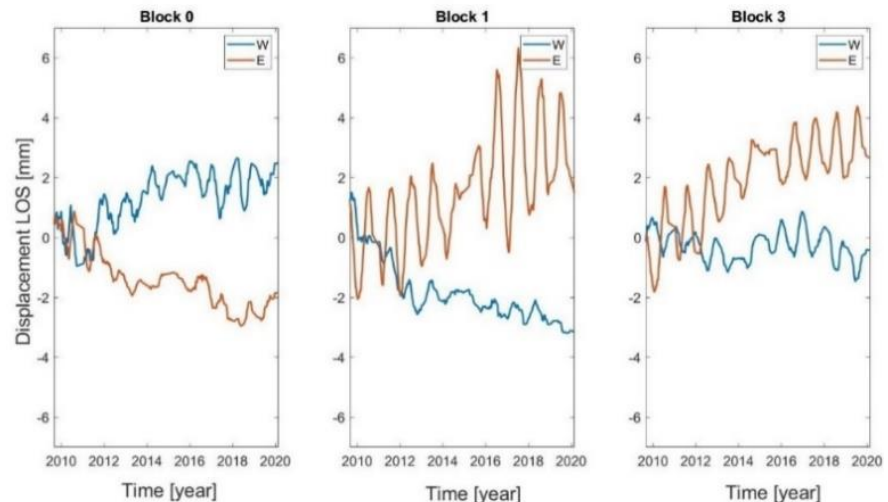

Figure 13. Via Farini bridge: TRX displacement time histories at the West (W) and East (E) of the deck for possibly damaged blocks. 


\section{$5.4 \quad$ Viale Monza 101 and 119 bridges}

Viale Monza 101 bridge is a $26 \mathrm{~m}$ long railway bridge that crosses a road. The deck is supported by two high concrete columns. The nearby Viale Monza 119 bridge is a 28 $\mathrm{m}$ long railway bridge that crosses the same road (Viale Monza) $150 \mathrm{~m}$ further North (see Figure 14). The double-deck structure rests on two long concrete columns. Both bridges are surrounded by buildings of similar height over the ground level. This condition makes the selection of the MPs located on the bridge more challenging. Moreover, due to the short length of the bridges' span, the RSAT dataset contains a very limited number of MPs after the preprocessing of data. For this reason, the comparison between the high and low spatial resolution datasets was not possible. The analysis of the InSAR data is carried out using only the TSX dataset available for the period between September 2010 and the beginning of January 2019 when maintenance interventions were carried out on both bridges.

In this case, all the MPs were supposed to be part of the same cluster and the average displacement was considered as the reference displacement for the bridge.

The mean LOS displacement of the area surrounding the two bridges has been calculated as considering the average of the MPs LOS displacements in a range of $200 \mathrm{~m}$ around the bridge. In the considered period of about 10 years, a total displacement of about $3 \mathrm{~mm}$ was measured.

The RD of the two bridges are compared in Figure 15 with the mean displacement of the surrounding area. The relevant Pearson correlation coefficients are equal to 0.97 (Viale Monza 101) and 0.98 (Viale Monza 119) for the two bridges which lead to attribute the displacements of the two bridges to a slow settlement of the soil.

For these two case studies, as expected for reinforced concrete bridges, the correlation between LOS displacements and temperature is negligible.

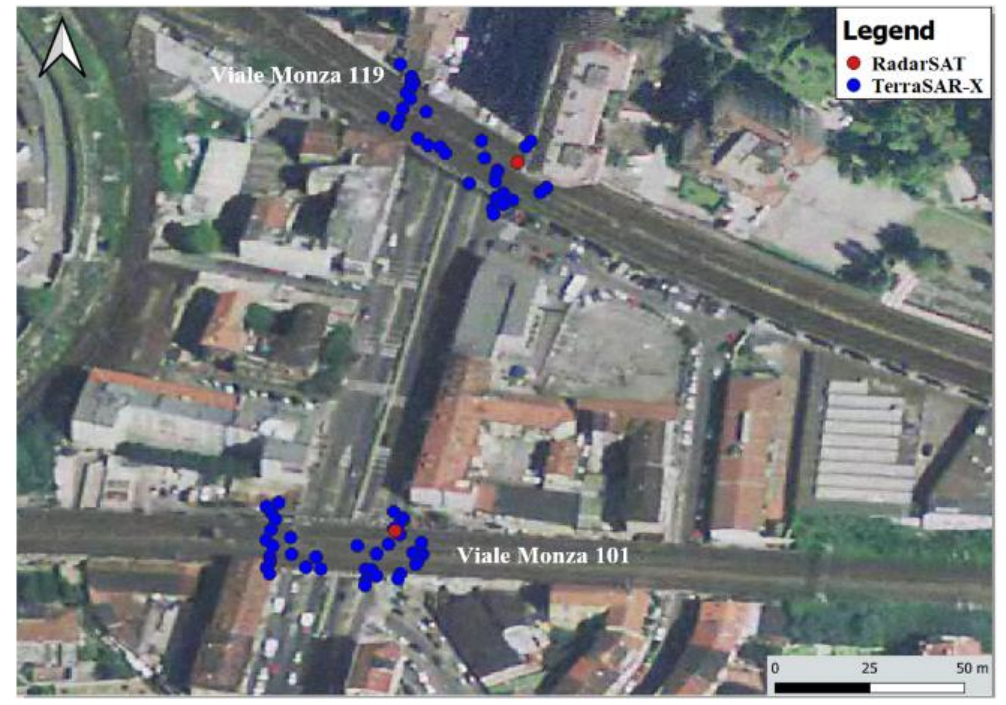

Figure 14. Viale Monza 101 and 119 bridges: A view from above with MPs detected by RSAT and TSX datasets. 


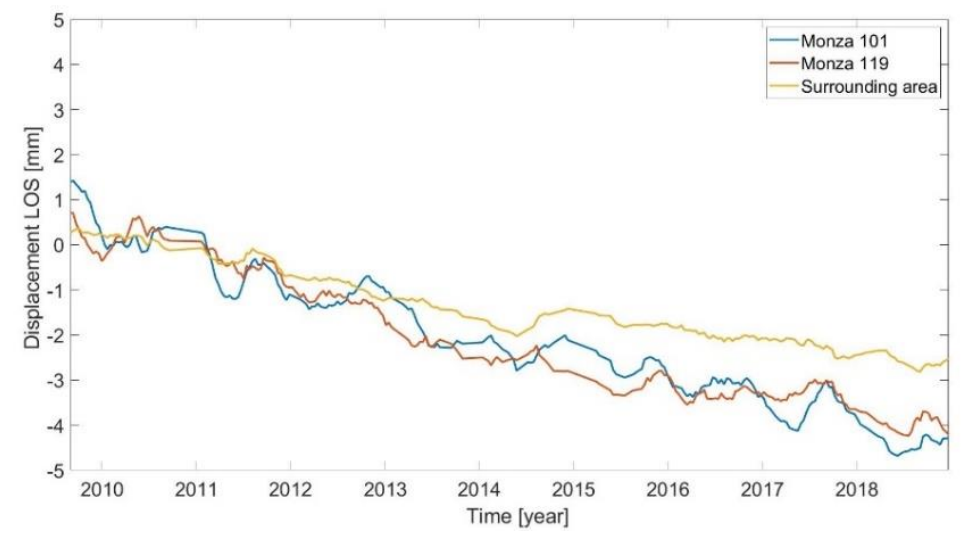

Figure 15. Viale Monza 101 and 119 bridges: Displacement time series of bridges' RP and the surrounding area.

\subsection{Discussion of results}

The analysis of InSAR data carried out according to the procedure outlined in Section 4 allowed to investigate the correlation of bridge displacements with thermal variations, with settlements of the area surrounding the bridge, and to highlight anomalies possibly related to structural changes.

The comparison of the results that can be obtained using high- or low- spatial resolution datasets shows that the use of the former enables not only to measure displacements with a higher resolution but, more importantly, to apply the InSAR technique to acquire information about short bridges that are prevalent in urban areas.

In the case studies considered, the comparison between low and high spatial resolution satellites was not always possible due to the lower number of MPs obtained with low resolution satellites. For the cases where the comparison was possible results were not always consistent. As an example, for the bridge in Via Lodovico il Moro 2 the low and high spatial resolution radars detect displacement peaks at similar times, but there is an important difference in the magnitude of the displacements which is likely due to the different radar bands and to a cycle slip issue, as explained in Section 5.2.

The use of InSAR in urban areas is also challenged by the presence of constructions around the bridge that might not only impede the radar beam to reach the bridge surface thereby reducing the number of MPs over the bridge deck, but can also prevent to distinguish the MPs located on the bridge deck from those located on the surrounding constructions. High spatial resolution and X-band satellites suite better for the monitoring of urban bridges because they ease and provide higher accuracy in MPs detection and positioning on the bridge.

The availability of high-resolution data also provides information about the influence of thermal variations on displacements and to study the relevant movements of the bridge when these are not negligible with respect to the accuracy of the sensor on the satellite and the InSAR technique. The results obtained on the case studies suggest a higher sensitivity of the X-band radar to temperature variation. 


\section{Conclusion}

This paper reports the outcomes of a study aimed to investigate the potentiality of the InSAR technology to detect anomalies in urban bridges. A procedure to process displacement data provided by this technique is proposed and applied to four bridges located in the Milan urban area using both high and low-spatial resolution radar data. Results show that high-resolution data enable to identify displacements correlated to thermal variations and trends in displacement time histories correlated with the settlement of the soil at a large scale or to anomalies in specific portions of the same bridge. This information provides interesting information to run possible screening procedures over large areas, aiming to select bridges requiring more in depth investigations. It should be noted that the dataset available in this study contained only data acquired along "descending orbits". More information would have been provided if both acquisition geometries (corresponding to ascending and descending acquisition modes) were available, since the computation of the displacements in the vertical and horizontal planes would have been possible together with a more clear interpretation of the bridge movements.

Further analysis will investigate how the integration of information from InSAR data with information from other sources, such as contact sensors, visual inspections, and design plans can improve the assessment of the structural condition and possibly allow the definition of displacement thresholds to use for the setup of an alarm procedure at large scale triggered by the InSAR data.

An interesting finding of this study is that MPs located in proximity of the railway show large negative displacements, which are attributed to movements of the railway ballast. These MPs should be carefully considered during the analysis since they might be wrongly associated to structural displacements.

The results reported in this paper show that the selection of MPs on the bridge must be carefully carried out. The availability of a Dense Digital Surface Model (DDSM) that enables an accurate 3D spatial description of the sensed object could help to improve the assignment of the MPs to the monitored structure

As a general comment, the use of the InSAR technology with high spatial resolution data can provide information useful to perform a large-scale survey on a large number of bridges, that may enable to detect anomalies. This information enables to trigger more refined investigations, carried out for example through visual inspections or nondestructive tests, on selected bridges for which an alert is provided by the InSAR data. It is worth recalling that the resolution and the temporal frequency of acquisition of satellite data is increasing steadily and sub-meter radar imagery, so far used for intelligence and security applications only, has now become available for commercial projects and civilian applications.

\section{References}

1. A. Schroten et al., "Overview of transport infrastructure expenditures and costs," Publications Office of the European Union, 2019. doi: 10.2832/853267. 
2. A. Occhiuzzi, "Consiglio Nazionale delle Ricerche." https://www.cnr.it/it/notastampa/n-8247/direttore-cnr-itc-sul-viadotto-morandi-di-genova.

3. M. P. Limongelli, E. Chatzi, M. Döhler, G. Lombaert, and E. Reynders, "Towards extraction of vibration-based damage indicators," 2016.

4. P. F. Giordano and M. P. Limongelli, "Response-based time-invariant methods for damage localization on a concrete bridge," Struct. Concr., vol. 21, no. 4, pp. 1254-1271, Aug. 2020, doi: 10.1002/suco.202000013.

5. P. F. Giordano, F. Ubertini, N. Cavalagli, A. Kita, and M. G. Masciotta, "Four years of structural health monitoring of the San Pietro bell tower in Perugia, Italy: two years before the earthquake versus two years after," Int. J. Mason. Res. Innov., vol. 5, no. 4, p. 445, 2020, doi: 10.1504/IJMRI.2020.111797.

6. M. Domaneschi, M. P. Limongelli, and L. Martinelli, "Damage detection in a suspension bridge model using the Interpolation Damage Detection Method," 2012, doi: 10.1201/b12352-452.

7. A. Ferretti, A. Fumagalli, F. Novali, C. Prati, F. Rocca, and A. Rucci, "A new algorithm for processing interferometric data-stacks: SqueeSAR," 2011, doi: 10.1109/TGRS.2011.2124465.

8. A. Hooper, P. Segall, and H. Zebker, "Persistent scatterer interferometric synthetic aperture radar for crustal deformation analysis, with application to Volcán Alcedo, Galápagos," J. Geophys. Res. Solid Earth, vol. 112, no. 7, pp. 1-21, 2007, doi: 10.1029/2006JB004763.

9. G. Barla, A. Tamburini, S. Del Conte, and C. Giannico, "InSAR monitoring of tunnel induced ground movements," Geomech. Tunn., vol. 9, no. 1, pp. 15-22, Feb. 2016, doi: 10.1002/geot.201500052.

10. C. A. Bischoff, A. Ferretti, F. Novali, A. Uttini, C. Giannico, and F. Meloni, "Nationwide deformation monitoring with SqueeSAR ${ }^{\circledR}$ using Sentinel-1 data," Proc. Int. Assoc. Hydrol. Sci., vol. 382, pp. 31-37, 2020, doi: 10.5194/piahs-382-31-2020.

11. E. J. Hoppe et al., "Deformation Monitoring of Posttensioned Bridges Using HighResolution Satellite Remote Sensing,” J. Bridg. Eng., 2019, doi: 10.1061/(asce)be.19435592.0001479

12. J. J. Sousa and L. Bastos, "Multi-temporal SAR interferometry reveals acceleration of bridge sinking before collapse," Nat. Hazards Earth Syst. Sci., vol. 13, no. 3, pp. 659667, Mar. 2013, doi: 10.5194/nhess-13-659-2013.

13. S. Selvakumaran, S. Plank, C. Geiß, C. Rossi, and C. Middleton, "Remote monitoring to predict bridge scour failure using Interferometric Synthetic Aperture Radar (InSAR) stacking techniques," Int. J. Appl. Earth Obs. Geoinf., vol. 73, pp. 463-470, Dec. 2018, doi: 10.1016/j.jag.2018.07.004.

14. P. Milillo, G. Giardina, D. Perissin, G. Milillo, A. Coletta, and C. Terranova, "PreCollapse Space Geodetic Observations of Critical," Remote Sens.

15. R. Lanari et al., "Comment on 'Pre-Collapse Space Geodetic Observations of Critical Infrastructure: The Morandi Bridge, Genoa, Italy' by Milillo et al. (2019)," Remote Sens., 2020, doi: 10.3390/rs12244011.

16. A. Pigorini, M. Ricci, and A. Sciotti, "La tecnica PSInSAR TM di telerilevamento satellitare applicata al progetto ed alla realizzazione delle infrastrutture ferroviarie Satellite remote-sensing PSInSAR TM technique applied to design and construction of 
railway infrastructures," no. 2, pp. 729-757, 2010.

17. F. Koudogbo, A. Urdiroz, J. G. Robles, and G. Chapron, "Radar interferometry as an innovative solution for monitoring the construction of the Grand Paris Express metro network - First results," Insa. Pap. Altamira, 2019.

18. G. Fornaro, D. Reale, and S. Verde, "Bridge Thermal Dilation Monitoring With Millimeter Sensitivity via Multidimensional SAR Imaging," IEEE Geosci. Remote Sens. Lett., vol. 10, no. 4, pp. 677-681, Jul. 2013, doi: 10.1109/LGRS.2012.2218214.

19. M. Lazecky, I. Hlavacova, M. Bakon, J. J. Sousa, D. Perissin, and G. Patricio, "Bridge Displacements Monitoring Using Space-Borne X-Band SAR Interferometry," IEEE J. Sel. Top. Appl. Earth Obs. Remote Sens., vol. 10, no. 1, pp. 205-210, Jan. 2017, doi: 10.1109/JSTARS.2016.2587778.

20. Q. Huang, M. Crosetto, O. Monserrat, and B. Crippa, "Displacement monitoring and modelling of a high-speed railway bridge using C-band Sentinel-1 data," ISPRS J. Photogramm. Remote Sens., vol. 128, pp. 204-211, Jun. 2017, doi: 10.1016/j.isprsjprs.2017.03.016.

21. L. Zhang, Q. Sun, and J. Hu, "Potential of TCPInSAR in monitoring linear infrastructure with a small dataset of SAR images: Application of the Donghai Bridge, China," Appl. Sci., vol. 8, no. 3, 2018, doi: 10.3390/app8030425.

22. M. Del Soldato, R. Tomás, J. Pont, G. Herrera, J. C. G. Lopez-Davalillos, and O. Mora, "A multi-sensor approach for monitoring a road bridge in the Valencia harbor (SE Spain) by SAR Interferometry (InSAR)," Rend. Online Soc. Geol. Ital., vol. 41, pp. 235238, 2016, doi: 10.3301/ROL.2016.137.

23. Q. Huang et al., "Displacement Monitoring and Health Evaluation of Two Bridges Using Sentinel-1 SAR Images," Remote Sens., vol. 10, no. 11, p. 1714, Oct. 2018, doi: 10.3390/rs10111714.

24. R. Tomás et al., "Monitoring an earthfill dam using differential SAR interferometry: La Pedrera dam, Alicante, Spain," Eng. Geol., vol. 157, pp. 21-32, May 2013, doi: 10.1016/j.enggeo.2013.01.022.

25. W. Zhou, S. Li, Z. Zhou, and X. Chang, "Remote Sensing of Deformation of a High Concrete-Faced Rockfill Dam Using InSAR: A Study of the Shuibuya Dam, China," Remote Sens., vol. 8, no. 3, p. 255, Mar. 2016, doi: 10.3390/rs8030255.

26. D. Di Martire et al., "Comparison between Differential SAR interferometry and ground measurements data in the displacement monitoring of the earth-dam of Conza della Campania (Italy)," Remote Sens. Environ., 2014, doi: 10.1016/j.rse.2014.03.014.

27. S. Arangio, F. Calò, M. Di Mauro, M. Bonano, M. Marsella, and M. Manunta, "An application of the SBAS-DInSAR technique for the assessment of structural damage in the city of Rome," Struct. Infrastruct. Eng., vol. 10, no. 11, pp. 1469-1483, Nov. 2014, doi: 10.1080/15732479.2013.833949.

28. N. Cavalagli, A. Kita, S. Falco, F. Trillo, M. Costantini, and F. Ubertini, "Satellite radar interferometry and in-situ measurements for static monitoring of historical monuments: The case of Gubbio, Italy," Remote Sens. Environ., vol. 235, p. 111453, Dec. 2019, doi: 10.1016/j.rse.2019.111453.

29. D. Cusson, K. Trischuk, D. Hébert, G. Hewus, M. Gara, and P. Ghuman, "SatelliteBased InSAR Monitoring of Highway Bridges: Validation Case Study on the North Channel Bridge in Ontario, Canada," Transp. Res. Rec. J. Transp. Res. Board, vol. 2672, 
no. 45, pp. 76-86, Dec. 2018, doi: 10.1177/0361198118795013.

30. A. Ferretti, C. Prati, and F. Rocca, "Permanent scatterers in SAR interferometry," IEEE Trans. Geosci. Remote Sens., 2001, doi: 10.1109/36.898661.

31. J. M. D. Blasco, M. Foumelis, C. Stewart, and A. Hooper, "Measuring urban subsidence in the Rome Metropolitan Area (Italy) with Sentinel-1 SNAP-StaMPS Persistent Scatterer Interferometry," Remote Sens., 2019, doi: 10.3390/rs11020129.

32. "QGIS," 2020. https://www.qgis.org/it/site/.

33. "Geoportale Regione Lombardia." http://www.geoportale.regione.lombardia.it/.

34. “ARPA Lombardia.” https://www.arpalombardia.it/Pages/ARPA_Home_Page.aspx.

35. K. Pearson, "VII. Note on regression and inheritance in the case of two parents," Proc.

R. Soc. London, vol. 58, no. 347-352, pp. 240-242, Dec. 1895, doi: 10.1098/rspl.1895.0041. 